\title{
Long-term combination chemotherapy using eribulin and trastuzumab for three patients with human epidermal growth factor receptor 2-positive metastatic breast cancer
}

\author{
Toshiaki Saeki ${ }^{1}$ Kazuhiro Araki ${ }^{2}$ Ken Shimada $^{3}$ 'Takashi Shigekawa ${ }^{1}$. \\ Hirofumi Nakayama ${ }^{4} \cdot$ Yoshihiko Segawa $^{5} \cdot$ Hirofumi Mukai $^{6}$
}

Received: 14 February 2016/ Accepted: 24 May 2016/Published online: 21 June 2016

(c) The Author(s) 2016. This article is published with open access at Springerlink.com

\begin{abstract}
The combination chemotherapy regimen of eribulin (ERI) and trastuzumab (TRA) - the ERI-TRA regimen-has been shown to be highly tolerable for patients with recurrent or metastatic human epidermal growth factor receptor 2 (HER2)-positive breast cancer. However, no sufficient clinical evidence is available for the long-term safety profile of the regimen. We report on three patients in the Phase I combination study of the regimen, for whom the regimen could be conducted over the long term. Patient \#1 was a 68 -year-old woman and underwent the regimen until cycle 23. Patient \#2 was a 61-year-old woman and underwent the regimen until cycle 27. Patient \#3 was a 59-year-old woman and underwent the regimen until cycle 22. All these patients had undergone TRA-based
\end{abstract}

Toshiaki Saeki

tsaeki@saitama-med.ac.jp

1 Department of Breast Oncology, International Medical Center, Saitama Medical University, 1397-1, Yamane, Hidaka, Saitama 350-1298, Japan

2 Breast Medical Oncology, Breast Oncology Center, The Cancer Institute Hospital of JFCR, 3-8-31, Ariake, Koto-ku, Tokyo 135-0141, Japan

3 Division of Medical Oncology, Department of Medicine, Showa University Koto Toyosu Hospital, 5-1-38, Toyosu, Koto-Ku, Tokyo 135-8577, Japan

4 Division of Medical Oncology, Department of Medicine, Showa University Fujigaoka Hospital, 1-30, Fujigaoka, Aoba-ku, Yokohama, Kanagawa 227-8501, Japan

5 Department of Medical Oncology, International Medical Center, Saitama Medical University, 1397-1, Yamane, Hidaka, Saitama 350-1298, Japan

6 Department of Breast and Medical Oncology, National Cancer Center Hospital East, 6-5-1, Kashiwanoha, Kashiwa, Chiba 277-8577, Japan combination therapy before the onset of the regimen. Any new categories of adverse events did not occur in association with the long-term combination chemotherapy. Neutropenia experienced by these patients was reversible and easily manageable by dose adjustment (interruption/ delay and reduction). Neither increase in the risk of cardiomyopathy nor the worsening of peripheral neuropathy greater than grade 1 was found. The present regimen was suggested to be a novel chemotherapeutic option for patients with HER2-positive recurrent or metastatic breast cancer. The fact that the long-term ERI-TRA regimen was successfully conducted for these patients can be supplementary clinical information that is beneficial for clinical oncologists.

Keywords Breast cancer - Eribulin · Trastuzumab · Anticancer drug $\cdot$ Combination chemotherapy

\section{Introduction}

We conducted a Phase I combination study of eribulin (ERI) mesylate and trastuzumab (TRA) in 12 Japanese female patients with HER2-positive metastatic breast cancer [1]. The design of the study consisted of Part 1 (weekly administration of TRA) and Part 2 (triweekly administration of TRA). In both Part 1 and 2, ERI mesylate $1.4 \mathrm{mg} / \mathrm{m}^{2}$ [equivalent to ERI $1.23 \mathrm{mg} / \mathrm{m}^{2}$ (expressed as free base)] was administered intravenously for $2-5 \mathrm{~min}$ on days 1 and 8 of each cycle. In Part 1, TRA $4 \mathrm{mg} / \mathrm{kg}$ was given by $\geq 90$-minute infusion as the loading dose, followed by $\geq 30$-minute infusion at $2 \mathrm{mg} / \mathrm{kg}$ on days 1,8 , and 15 of the 21-day cycle. In Part 2, TRA $8 \mathrm{mg} / \mathrm{kg}$ was administered as the loading dose, followed by infusion at $6 \mathrm{mg} / \mathrm{kg}$ on day 1 of the second and subsequent 21-day 
cycles [1]. Consequently, the combination chemotherapy regimen of ERI mesylate $1.4 \mathrm{mg} / \mathrm{m}^{2}$ on days 1 and 8 of the 21-day cycle with weekly or triweekly TRA was shown to be highly tolerable. Results from the Phase I combination study [1] and the Phase II study [2] suggest the reasonableness of further examining the long-term safety profile of the regimen.

We hereby report on three Japanese patients with HER2positive metastatic breast cancer for whom this regiment was continued after the cutoff date (August 2, 2013) in the Phase I combination study and in whom subsequent 9-month follow-up revealed that the total number of the chemotherapy cycles had exceeded 20.

\section{Case reports}

Patient \#1: A 68-year-old woman in Part 1. The patient had undergone mastectomy for right breast cancer at our hospital and was enrolled in this study because of progressive disease (PD). Immunohistochemistry (IHC) staining revealed HER2 positivity (3+), as well as estrogen receptor (ER) and progesterone receptor (PgR) negativity. The patient had been treated with a combination of tegafur and tamoxifen as anticancer drugs for adjuvant chemotherapy, followed by a combination of TRA and paclitaxel (PTX) for the treatment of breast cancer that recurred. At baseline, the patient had grade 1 peripheral neuropathy but did not present cardiac dysfunction. After enrollment, dose-limiting toxicity (DLT) did not develop. The unequivocal progression of nontarget lesions in the right femur led to treatment discontinuation in cycle 23. Grade 2 or greater adverse events (AEs) in this study included grade 4 neutropenia, grade 3 leukopenia, grade 2 mucositis, grade 2 phlebitis, grade 2 anorexia, grade 2 dysgeusia, and grade 2 alopecia. Reduced left ventricular ejection fraction (LVEF) was not found.

Patient \#2: A 61-year-old woman in Part 1. The patient had undergone conservation surgery of the left breast at our hospital and was enrolled in this study because of PD. IHC staining revealed ER and PgR negativity, as well as HER 2 positivity $(2+)$ that was subsequently verified by gene amplification by fluorescence in situ hybridization (FISH). The patient had been treated by combination chemotherapy with doxorubicin (DXR) and cyclophosphamide, followed by PTX alone, radiotherapy, and TRA alone. Furthermore, the patient underwent combination chemotherapy with TRA and docetaxel (DTX) for the treatment of breast cancer that recurred. At baseline, the patient had grade 1 peripheral neuropathy but did not present cardiac dysfunction. After enrollment, DLT did not develop. The unequivocal progression of nontarget lesions in the right kidney led to treatment discontinuation in cycle 27. Grade 2 or greater AEs in this study included grade 4 neutropenia, grade 2 leukopenia, grade 2 extravascular leakage at injection site, grade 2 LVEF, grade 2 alopecia, and grade 2 hyperlipidemia. Grade 2 LVEF developed in cycle 19.

Patient \#3: A 59-year-old woman in Part 2. The patient could not be treated surgically because of stage IIIc breast cancer and was referred to our hospital for enrollment in this study. IHC staining revealed ER and PgR positivity, as well as HER2 positivity ( $3+$ ) that was subsequently verified by FISH. The patient had been treated by combination chemotherapy with TRA and PTX, followed by TRA alone. At baseline, the patient had grade 1 peripheral neuropathy but did not present cardiac dysfunction. After enrollment, DLT did not develop. Grade 2 or greater AEs in this study included grade 4 neutropenia, grade 3 febrile neutropenia, grade 3 hypertriglyceridemia, grade 2 leukopenia, grade 2 lymphopenia, grade 2 anemia, grade 2 pyrexia, grade 2 spondylolisthesis, grade 2 stomatitis, and grade 2 urticaria. Treatment was discontinued due to interstitial pneumonia in cycle 22. Reduced LVEF was not found. Patient characteristics at baseline are shown in Table 1.

The Phase I combination study described two patients with grade 2 reduced LVEF and indicated no clearly increased risk of severe cardiac dysfunction resulting from the present combination chemotherapy regimen because they recovered within 1 week without requiring treatment. The follow-up subsequent to the cutoff date for the Phase I combination study did not disclose any new case of reduced LVEF (Table 2).

\section{Discussion}

At the onset of the Phase I combination study in 2011, the preferred first-line chemotherapy regimens recommended by the National Comprehensive Cancer Network (NCCN) [3] - which combined TRA with one and/or two anticancer drugs, e.g., PTX [4, 5], DTX [6-10], capecitabine [CAPE] $[7,11,12]$, and vinorelbine [VNR] $[10,13,14]$-were conducted for patients with HER2-positive recurrent or metastatic breast cancer. The package inserts of these anticancer drugs describe the following as major AEs: (1) TRA: cardiomyopathy and anaphylactoid symptoms; (2) PTX: myelosuppression, hypersensitive reaction, and peripheral neuropathy; (3) DTX: myelosuppression, anaphylaxis, and nail disorders; (4) CAPE: dehydration, handfoot syndrome, and hepatopathy; and (5) VNR: myelosuppression, interstitial pneumonia, pulmonary congestion, and phlebitis. Furthermore, myelosuppression, infections, peripheral neuropathy, and hepatic dysfunction have been reported regarding ERI. 
Table 1 Patient characteristics at baseline

\begin{tabular}{|c|c|c|c|}
\hline \multirow[t]{2}{*}{ Variables } & \multicolumn{3}{|l|}{ Patients } \\
\hline & $N=3 *$ & $N=9^{\dagger}$ & Total $=12$ \\
\hline ER and/or PgR plus disease, $n(\%)$ & $1(33.3)$ & $4(44.4)$ & $5(41.7)$ \\
\hline \multicolumn{4}{|l|}{ ECOG performance status, $n(\%)$} \\
\hline 0 & 0 & $5(41.7)$ & $5(41.7)$ \\
\hline 1 & $3(100)$ & $4(44.4)$ & $7(58.3)$ \\
\hline \multicolumn{4}{|c|}{ Number of prior chemotherapy regimens, $n(\%)^{*}$} \\
\hline 1 & $1(33.3)$ & 0 & $1(8.3)$ \\
\hline 2 & 0 & $1(11.1)$ & $1(8.3)$ \\
\hline 3 & $2(66.7)$ & 0 & $2(16.7)$ \\
\hline 4 & 0 & $2(22.2)$ & $2(16.7)$ \\
\hline$\geq 5$ & 0 & $6(66.7)$ & $6(50.0)$ \\
\hline Median (range) & $3.0(1-3)$ & $5.0(2-14)$ & $4.5(1-14)$ \\
\hline \multicolumn{4}{|c|}{ Number of prior trastuzumab treatment, $n(\%)^{*}$} \\
\hline 1 & $2(66.7)$ & $1(11.1)$ & $3(25.0)$ \\
\hline 2 & $1(33.3)$ & $3(33.3)$ & $4(33.3)$ \\
\hline$\geq 3$ & 0 & $5(55.6)$ & $5(41.7)$ \\
\hline Median (range) & $1.0(1-2)$ & $3.0(1-5)$ & $2.0(1-5)$ \\
\hline \multicolumn{4}{|l|}{ Prior taxane treatment, $n(\%)^{*}$} \\
\hline 1 & $2(66.7)$ & $5(55.6)$ & $7(58.3)$ \\
\hline 2 & $1(33.3)$ & $3(33.3)$ & $4(33.3)$ \\
\hline 3 & 0 & $1(11.1)$ & $1(8.3)$ \\
\hline Prior anthracycline treatment, $n(\%)$ & $1(33.3)$ & $5(55.6)$ & $6(50.0)$ \\
\hline
\end{tabular}

ECOG Eastern Cooperative Oncology Group, ER estrogen receptor, $P g R$ progesterone receptor

* Patients who received more than 20 cycles of the treatment

$\dagger$ Patients who received less than 20 cycles of the treatment

* Including the neoadjuvant, adjuvant, and therapeutic regimens
Neutropenia experienced by all 12 patients in the Phase I combination study was reversible and easily manageable by dose adjustment (interruption/delay and reduction). Three of 12 patients, in whom the long-term ERI-TRA regimen could be conducted, showed no increased risk of cardiomyopathy in association with the present regimen; nevertheless, reduced LVEF was observed with two patients prior to the cutoff date. Therefore, cardiac function should be routinely assessed in patients undergoing the ERI-TRA regimen, which is consistent with the recommendation for patients receiving trastuzumab in other combination therapies. Nonhematologic AEs, e.g., nausea, vomiting, pyrexia, and rash, were mild in intensity. Especially, it is of note that this regimen provoked little AEs that considerably affect the quality of life of patients during the chemotherapy cycles, e.g., hypersensitive reaction, nail disorders, phlebitis, as well as hand-foot syndrome observed with PTX, DTX, VNR, and CAPE, respectively. Prior studies of PTX and DTX have reported the incidences of grade $3 / 4$ peripheral neuropathy ranging between 5-35\% [15-17] and 3-14\% [18, 19]. Peripheral neuropathy was grade $1 / 2$ in all 12 patients, except one who had grade 3 peripheral neuropathy. Furthermore, any peripheral neuropathy that worsened to greater than grade 1 was not found in these three patients. At baseline, on the other hand, the patients presented the less number of prior chemotherapy regimens and less exposure to TRA as compared with the remaining nine patients; these facts probably favored the long-term management of the pathology. Examination of the safety profile revealed no emergence of any new categories of AEs associated with long-term combination chemotherapy subsequent to the cutoff date of Phase I combination study, which leads us to consider that the present regimen is safe for a long term. Taken together, we believe that information on the longterm safety of eribulin described above is of clinical relevance for clinical oncologists.

The objectives of treatment for patients with recurrent or metastatic breast cancer, about whom cure is hopeless, are the extension of their survival and the sustainment of or improvement in their quality of life. Therefore, it is essential to conduct anticancer combination chemotherapy for a longer period of time to fulfill these objectives. In many clinical settings, 
Table 2 Adverse events (all grades in $\geq 10 \%$ of patients and grade $3 / 4$ in total)

\begin{tabular}{|c|c|c|c|c|c|c|}
\hline \multirow[t]{3}{*}{ Preferred terms in CTCAE, $n(\%)$} & \multicolumn{6}{|l|}{ Patients } \\
\hline & \multicolumn{2}{|l|}{$N=3 *$} & \multicolumn{2}{|l|}{$N=9^{\dagger}$} & \multicolumn{2}{|l|}{ Total } \\
\hline & All grades & Grade 3/4 & All grades & Grade 3/4 & All grades & Grade 3/4 \\
\hline Leukopenia & $3(100.0)$ & $2(66.7)$ & $9(100.0)$ & $8(88.9)$ & $12(100.0)$ & $10(83.3)$ \\
\hline Neutropenia & $3(100.0)$ & $3(100.0)$ & $9(100.0)$ & $9(100.0)$ & $12(100.0)$ & $12(100.0)$ \\
\hline Anemia & $2(66.7)$ & 0 & $6(66.7)$ & 0 & $8(66.7)$ & 0 \\
\hline Lymphopenia & $1(33.3)$ & $1(33.3)$ & $2(22.2)$ & $2(22.2)$ & $3(25.0)$ & $3(25.0)$ \\
\hline Febrile neutropenia & $1(33.3)$ & $1(33.3)$ & $1(11.1)$ & $1(11.1)$ & $2(16.7)$ & $2(16.7)$ \\
\hline Palpitations & $1(33.3)$ & 0 & $1(11.1)$ & 0 & $2(16.7)$ & 0 \\
\hline Nausea & 0 & 0 & $3(33.3)$ & 0 & $3(25.0)$ & 0 \\
\hline Vomiting & $1(33.3)$ & 0 & $2(22.2)$ & 0 & $3(25.0)$ & 0 \\
\hline Constipation & $1(33.3)$ & 0 & $1(11.1)$ & 0 & $2(16.7)$ & 0 \\
\hline Stomatitis & $1(33.3)$ & 0 & $1(11.1)$ & 0 & $2(16.7)$ & 0 \\
\hline Pyrexia & $2(66.7)$ & 0 & $3(33.3)$ & 0 & $5(41.7)$ & 0 \\
\hline Malaise & $2(66.7)$ & 0 & $1(11.1)$ & 0 & $3(25.0)$ & 0 \\
\hline Chest discomfort & $1(33.3)$ & 0 & $1(11.1)$ & 0 & $2(16.7)$ & 0 \\
\hline Injection site reaction & $1(33.3)$ & 0 & $1(11.1)$ & 0 & $2(16.7)$ & 0 \\
\hline Lung infection & 0 & 0 & $2(22.2)$ & 0 & $2(16.7)$ & 0 \\
\hline Tonsillitis & $2(66.7)$ & 0 & 0 & 0 & $2(16.7)$ & 0 \\
\hline ALAT increased & $1(33.3)$ & 0 & $2(22.2)$ & 0 & $3(25.0)$ & 0 \\
\hline ASAT increased & $1(33.3)$ & 0 & $2(22.2)$ & 0 & $3(25.0)$ & 0 \\
\hline Blood CPK increased & $1(33.3)$ & 0 & $2(22.2)$ & 0 & $3(25.0)$ & 0 \\
\hline Ejection fraction decreased ${ }^{\S}$ & $1(33.3)$ & 0 & $1(11.1)$ & 0 & $2(16.7)$ & 0 \\
\hline Decreased appetite & $3(100.0)$ & 0 & $3(33.3)$ & 0 & $6(50.0)$ & 0 \\
\hline Hypertriglyceridemia & $1(33.3)$ & $1(33.3)$ & $2(22.2)$ & $2(22.2)$ & $3(25.0)$ & $3(25.0)$ \\
\hline Hypophosphatemia & 0 & 0 & $1(11.1)$ & $1(11.1)$ & $1(8.3)$ & $1(8.3)$ \\
\hline Myalgia & $2(66.7)$ & 0 & $2(22.2)$ & 0 & $4(33.3)$ & 0 \\
\hline Muscle spasms & $1(33.3)$ & 0 & $1(11.1)$ & 0 & $2(16.7)$ & 0 \\
\hline Dysgeusia & $2(66.7)$ & 0 & $2(22.2)$ & 0 & $4(33.3)$ & 0 \\
\hline Peripheral neuropathy* & $2(66.7)$ & 0 & $3(33.3)$ & $1(11.1)$ & $5(41.7)$ & $1(8.3)$ \\
\hline Headache & $1(33.3)$ & 0 & $1(11.1)$ & 0 & $2(16.7)$ & 0 \\
\hline Alopecia & $3(100.0)$ & NA & $5(55.6)$ & NA & $8(66.7)$ & NA \\
\hline Rash & $2(66.7)$ & 0 & $3(33.3)$ & 0 & $5(41.7)$ & 0 \\
\hline
\end{tabular}

ALAT alanine aminotransferase, ASAT aspartate aminotransferase, $C P K$ creatinine phosphokinase, NA not applicable, CTCAE Common terminology criteria for adverse events, Japanese version 4.0

* Patients who received more than 20 cycles of the treatment

$\dagger$ Patients who received 20 or less cycles of the treatment

* Including neuropathy peripheral and peripheral sensory neuropathy in preferred terms

$\S$ Patient \#2 developed this change in cycle 19

however, we frequently find treatment difficult to continue because of disease progression, sorts of AEs, loss of efficacy, and others. In fact, the ERI-TRA regimen was discontinued not later than cycle 12 in nine of our 12 patients [1]. The 2015 NCCN Clinical Practice Guidelines in Oncology: Breast Cancer mention two preferred first-line regimens for chemotherapy combinations: pertuzumab (PER), TRA, and DTX; and PER,
TRA, and PTX, in addition to other first-line regimens combining TRA with PTX \pm carboplatin, DTX, CAPE, or VNR [20]. The results from our analysis indicated no deterioration of hematologic or nonhematologic AEs in three patients of interest, suggesting the potential of the present regimen as a novel chemotherapeutic option for patients with HER2-positive recurrent or metastatic breast cancer. 
The fact that long-term chemotherapy using ERI mesylate in combination with TRA was successfully conducted for patients with recurrent or metastatic HER2positive breast cancer can be supplementary clinical information that is beneficial for clinical oncologists.

Acknowledgments Funding to support this study was provided by Eisai Co., Ltd. The authors thank Satoshi Sakima, MD, for his gracious review of the manuscript.

\section{Compliance with ethical standards}

Conflict of interest statement Toshiaki Saeki received lecture fees and research funding from Eisai Co., Ltd. Kazuhiro Araki received research funding from Eisai Co., Ltd. for another study. Yoshihiko Segawa received lecture fees from Eisai Co., Ltd. and Chugai Pharmaceutical Co., Ltd., and received research funding from Chugai Pharmaceutical Co., Ltd. Hirofumi Mukai received lecture fees and research funding from Eisai Co., Ltd. Other authors have no conflict of interest to disclose.

Ethical approval All procedures performed in studies involving human participants were in accordance with the ethical standards of the institutional and/or national research committee and with the 1964 Helsinki declaration and its later amendments or comparable ethical standards.

Informed consent Informed consent was obtained from all individual participants included in the study.

Open Access This article is distributed under the terms of the Creative Commons Attribution 4.0 International License (http://crea tivecommons.org/licenses/by/4.0/), which permits unrestricted use, distribution, and reproduction in any medium, provided you give appropriate credit to the original author(s) and the source, provide a link to the Creative Commons license, and indicate if changes were made.

\section{References}

1. Mukai H, Saeki T, Shimada K et al (2015) Phase 1 combination study of Eribulin mesylate with trastuzumab for advanced or recurrent human epidermal growth factor receptor 2 positive breast cancer. Invest New Drugs 33:119-127

2. Wilks S, Puhalla S, O'Shaughnessy J et al (2014) Phase 2, multicenter, single-arm study of eribulin mesylate with trastuzumab as first-line therapy for locally recurrent or metastatic HER2-positive breast cancer. Clin Breast Cancer 14:405-412

3. Carlson RW, Allred DC, Anderson BO et al (2011) Invasive breast cancer. J Natl Compr Canc Netw 9:136-222

4. Slamon DJ, Leyland-Jones B, Shak S et al (2001) Use of chemotherapy plus a monoclonal antibody against HER2 for metastatic breast cancer that overexpresses HER2. N Engl J Med 344:783-792

5. Gori S, Colozza M, Mosconi AM et al (2004) Phase II study of weekly paclitaxel and trastuzumab in anthracycline- and taxanepretreated patients with HER2-overexpressing metastatic breast cancer. Br J Cancer 90:36-40

6. Swain SM, Kim SB, Cortés J et al (2013) Pertuzumab, trastuzumab, and docetaxel for HER2-positive metastatic breast cancer
(CLEOPATRA study): overall survival results from a randomised, double-blind, placebo-controlled, phase 3 study. Lancet Oncol 14:461-471

7. Wardley AM, Pivot X, Morales-Vasquez F et al (2010) Randomized phase II trial of first-line trastuzumab plus docetaxel and capecitabine compared with trastuzumab plus docetaxel in HER2-positive metastatic breast cancer. J Clin Oncol 28:976-983

8. Marty M, Cognetti F, Maraninchi D et al (2005) Randomized phase II trial of the efficacy and safety of trastuzumab combined with docetaxel in patients with human epidermal growth factor receptor 2-positive metastatic breast cancer administered as firstline treatment. J Clin Oncol 23:4265-4274

9. Valero V, Forbes J, Pegram MD et al (2011) Multicenter phase III randomized trial comparing docetaxel and trastuzumab with docetaxel, carboplatin, and trastuzumab as first-line chemotherapy for patients with HER2-gene-amplified metastatic breast cancer (BCIRG 007 study): two highly active therapeutic regimens. J Clin Oncol 29:149-156

10. Livingston RB, Barlow WE, Kash JJ et al (2011) SWOG S0215: a phase II study of docetaxel and vinorelbine plus filgrastim with weekly trastuzumab for HER2-positive, stage IV breast cancer. Breast Cancer Res Treat 130:123-131

11. Schaller G, Fuchs I, Gonsch T et al (2007) Phase II study of capecitabine plus trastuzumab in human epidermal growth factor receptor 2 overexpressing metastatic breast cancer pretreated with anthracyclines or taxanes. J Clin Oncol 25:3246-3250

12. Chan A, Conte PF, Petruzelka L et al (2013) Phase II study of a triple combination of oral vinorelbine, capecitabine and trastuzumab as first-line treatment in HER2-positive metastatic breast cancer. Anticancer Res 33:2657-2664

13. Andersson M, Lidbrink E, Bjerre K et al (2011) Phase III randomized study comparing docetaxel plus trastuzumab with vinorelbine plus trastuzumab as first-line therapy of metastatic or locally advanced human epidermal growth factor receptor 2-positive breast cancer: the HERNATA Study. J Clin Oncol 29:264-271

14. Esfahani K, Ferrario C, Le P, Panasci L (2014) The trastuzumab and vinorelbine combination: an alternative to taxane-based chemotherapy for early-stage and locally advanced her2-positive breast cancer. Curr Oncol 21:e723-e727

15. Lee JJ, Swain SM (2006) Peripheral neuropathy induced by microtubule-stabilizing agents. J Clin Oncol 24:1633-1642

16. Seidman AD, Berry D, Cirrincione C et al (2008) Randomized phase III trial of weekly compared with every-3-weeks paclitaxel for metastatic breast cancer, with trastuzumab for all HER-2 overexpressors and random assignment to trastuzumab or not in HER-2 nonoverexpressors: final results of Cancer and Leukemia. J Clin Oncol 26:1642-1649

17. Winer EP, Berry DA, Woolf S, Duggan D et al (2004) Failure of higher-dose paclitaxel to improve outcome in patients with metastatic breast cancer: cancer and leukemia group B trial 9342 . J Clin Oncol 22:2061-2068

18. Stubblefield MD, Burstein HJ, Burton AW et al (2009) NCCN task force report: management of neuropathy in cancer. J Natl Compr Canc Netw Suppl 5:S1-S26

19. Jones SE, Erban J, Overmoyer B et al (2005) Randomized phase III study of docetaxel compared with paclitaxel in metastatic breast cancer. J Clin Oncol 23:5542-5551

20. NCCN Clinical Practice Guidelines in Oncology. Breast cancer. Version 2. 2015. http://www.nccn.org/professionals/physician gls/pdf/breast.pdf. Accessed April 2015 